\title{
ON TOPOLOGICAL ALGEBRA SHEAVES
}

\author{
ATHANASIOS KYRIAZIS
}

(Received 2 May 1991; revised 4 November 1994)

Communicated by J. H. Rubinstein

\begin{abstract}
Given two topological algebra sheaves, we seek that their tensor product be an (algebra) sheaf of the same type. We further study the latter sheaf in connection with the set of morphisms which are defined on it. As an application, we finally consider fundamental notions and results related to algebras of holomorphic functions in the framework of topological algebra sheaves.

1990 Mathematics subject classification (Amer. Math. Soc.): primary 46M20, 46M05; secondary 32D10, $32 \mathrm{~F} 99$.

Keywords and phrases: Topological algebra sheaf, topological geometric space, tensor product algebra sheaf, structure sheaf envelope, Dirac transform, central morphism.
\end{abstract}

\section{Introduction}

Topological algebra sheaves have already been applied to study global properties of holomorphic functions (see, for example, [20, 21]). By a topological algebra sheaf we mean a sheaf whose local sections have the structure of a topological algebra (not necessarily normed). Moreover, we usually consider, with [20], a similar algebraictopological structure on the corresponding stalks (thus a non-trivial topology!).

In this paper we are concerned, more generally, with topological $\mathscr{A}$-algebra sheaves, viz. topological algebra sheaves for which the 'domain of coefficients' is another topological algebra sheaf $\mathscr{A}$ instead of (the constant sheaf) $\mathbb{C}$ of the complexes. By extending standard terminology (see for example [21, 22]), we call a topological geometric $\mathscr{A}$-space a pair $(X, \mathscr{E})$ consisting of a sheaf $\mathscr{E}$ as above over the topological space $X$. This might be viewed, in effect, as an abstract notion of a complex analytic space, the kind of sheaves considered being actually suggested by applications in abstract complex analysis (see for instance [23]), as well as in (finite or

(C) 1996 Australian Mathematical Society 0263-6115/96 \$A2.00+0.00 
infinite dimensional) differential geometry (see for example I. M. Gel'fand-KahzdanD. B. Fuks). An analogous study, but in a different setting, via the theory of natural function algebras, has been given already by C. E. Rickart [24].

More precisely, we are mainly interested in studying tensor product algebra sheaves. Thus, given two topological $\mathscr{A}$-algebra sheaves $\mathscr{E}, \mathscr{F}$, we consider their tensor product $\mathscr{E} \hat{\otimes}_{\mathscr{A}}^{\tau} \mathscr{F}$, as a sheaf generated by the corresponding tensor product $\mathscr{A}$-algebra presheaf (cf. (2.4)). In particular, we exhibit conditions making the latter presheaf into a topological $\mathscr{A}$-algebra sheaf (see Examples 2.1 ) the so-called tensor product $\mathscr{A}$-algebra sheaf. As a first application, one gets a sheaf-theoretic version of the basic relation connecting the generalized spectrum of a topological tensor product algebra with the spectra of the factor algebras, thus obtaining a broad generalization of previous similar results (cf. $[6,14,16,19])$.

Moreover, by extending the classical concept of envelope of holomorphy of a complex analytic manifold $(X, \mathscr{O})$ as the (numerical) spectrum of an appropriate topological algebra (cf. $[9,10])$, we consider the structure sheaf envelope of a geometric $\mathscr{A}$-space $(X, \mathscr{S})$ (cf. (4.1)). In particular, we give the connection between the structure sheaf envelopes of a suitable subspace of $\left(X, \mathscr{E} \hat{\otimes}_{\mathscr{A}} \mathscr{F}\right)$ and those of the factor sheaves (cf. Proposition 4.1 and Corollary 4.1). These results are further extended to generalized structure sheaf envelopes, exploiting a systematic use of topological geometric $\mathscr{A}$-spaces and generalized spectra of topological algebras (cf. Theorem 5.1).

Finally, as another application of the preceding, one obtains, within this framework, a kind of 'Dirac transform'. This leads to the notion of a suitable continuous $\mathbb{A}$ morphism (central $\mathbb{A}$-morphism, with $\mathbb{A}$ a constant topological algebra sheaf) of the topological algebra of germs of sections of a topological tensor product $\mathbb{A}$-algebra sheaf (Proposition 6.1, Theorem 6.1). Yet this discussion exemplifies the rôle of the 'spectrum functor' in the finite or infinite dimensional holomorphy.

I wish to express my sincere thanks to Professor A. Mallios for several stimulating and helpful discussions, as well as for his encouragement and interest during the writing of this work.

\section{Topological algebra sheaves. Preliminaries}

The topological vector spaces and algebras considered are over the complex numbers $\mathbb{C}$ and will be assumed Hausdorff.

Thus, given a topological algebra $\mathbb{A}$, an $\mathbb{A}$-algebra (resp. $\mathbb{A}$-module, cf. [1, Chapter II, §1.1; Chapter III, §1.1]) $E$ is called a topological A-algebra (resp. topological $\mathbb{A}$-module), whenever the 'action' of $\mathbb{A}$ on $E$ is a (jointly) continuous map.

Let $X$ be a topological space. By a topological algebra sheaf on $X$, we mean a 
topological algebra presheaf on $X$ (cf. [20]), which is 'complete' (cf. (S1), (S2) of [2, pp. 5, 6]). On the other hand, a sheaf $\mathscr{E}$ generated by a topological algebra presheaf on $X$ is also a topological algebra sheaf on $X$ such that the corresponding algebra of local sections of $\mathscr{E}(\Gamma(U, \mathscr{E}) \equiv \mathscr{E}(U), U \subseteq X$ open) are, in fact, topological algebras (cf. [22]), hence, a topological algebra sheaf in the previous sense. In the sequel we will not distinguish between a topological algebra sheaf $\mathscr{E}$ on $X$ and the respective topological algebra (pre)sheaf of sections $\{\mathscr{E}(U)\}$, by writing $\mathscr{E}=\{\mathscr{E}(U)\}$.

Given a topological algebra sheaf $\mathscr{A}(=\{\mathscr{A}(U)\})$ on $X$, by a topological $\mathscr{A}$-algebra sheaf on $X$, we mean a topological algebra sheaf $\mathscr{E}(=\{\mathscr{E}(U)\})$ on $X$, with $\mathscr{E}(U)$ a topological $\mathscr{A}(U)$-algebra such that the appropriate compatibility conditions of the respective restriction maps are fulfilled. Locally (m-) convex, Fréchet, commutative $\mathscr{A}$-algebra sheaves with continuous multiplications, identity elements, (locally) equicontinuous spectra etc. will have the obvious meaning referring to the same properties of the corresponding algebra presheaves. Thus, the sheaf $\mathscr{C}=\{\mathscr{C}(U)\}$ of continuous $\mathbb{C}$-valued maps is a locally m-convex $\mathscr{C}^{\infty}=\left\{\mathscr{C}^{\infty}(U)\right\}$ (resp. $\mathscr{O}=\{\mathscr{O}(U)\}-$ )algebra sheaf on a $\mathscr{C}^{\infty}$-manifold (resp. complex analytic manifold) $X$. Here $\mathscr{C}^{\infty}(U)$ (resp. $\mathscr{O}(U)$ ) stands for the locally m-convex algebra of $\mathbb{C}$-valued differentiable (resp. holomorphic) maps on an open $U \subseteq X$ (cf. [19, Chapter IV, §4]).

Throughout the sequel the 'sheaf of coefficients' $\mathscr{A}$ will be understood to be a commutative algebra sheaf on $X$.

For any topological algebra sheaf $\mathscr{E}$ on $X$ and $S \subseteq X$, one defines the topological algebra of germs of (local) sections of $\mathscr{E}$ over $S$ by the relation

$$
\mathscr{E}(S):=\lim _{U \supseteq \mathfrak{S}} \mathscr{E}(U)
$$

where $U$ ranges over a fundamental system of open neighborhoods of $S$.

(Warning: $\mathscr{E}(S) \neq \Gamma(S, \mathscr{E})$ ). Furthermore $\mathscr{E}(S)$ is endowed with the inductive limit (vector space) topology such that $\mathscr{E}(S)$ is actually a topological algebra (cf. [19, Chapter IV, Lemma 2.2]). In particular, if $\mathscr{E}$ is a topological $\mathscr{A}$-algebra sheaf, then $\mathscr{E}(S)$ is a topological $\mathscr{A}(S)\left(:=\lim _{\vec{U}} \mathscr{A}(U)\right)$-algebra (cf. [16, §1]). In particular, the stalks of the previous sheaves (that is, $S=\{x\}, x \in X$ ) are given by

$$
\mathscr{E}_{x}:=\lim _{U \vec{U} \mathfrak{E}} \mathscr{E}(U),
$$

the latter set being, in fact (cf. (1.1)), a topological $\mathscr{A}_{x}\left(:=\lim _{\overrightarrow{U \ni x}} \mathscr{A}(U)\right)$-algebra.

A morphism $h: \mathscr{E} \rightarrow \mathscr{F}$ of topological $\mathscr{A}$-algebra sheaves is defined in the natural manner. As a consequence, the maps between the corresponding stalks $h_{x}:=$ $\lim _{U \rightarrow} h_{U}: \mathscr{E}_{x} \rightarrow \mathscr{F}_{x}$ are continuous $\mathscr{A}_{x}$-morphisms (cf. also [19, Chapter IV, §2] and $[16, \S 1])$. 


\section{The tensor product algebra sheaf}

Our aim in this section is to define the topological (tensor product) $\mathscr{A}$-algebra sheaf $\mathscr{E} \hat{\otimes}_{\mathscr{A}}^{\tau} \mathscr{F}$. However, we have first to digress by commenting on tensor product topological $\mathbb{A}$-algebras (for details cf. [14, 16]).

If $E, F$ are topological $\mathbb{A}$-algebras, by a compatible $\mathbb{A}$-topology on $E \otimes_{\mathbb{A}} F$, we mean a (Hausdorff) topology $\tau$ such that

the pair $\left(E \otimes_{\mathscr{A}} F, \tau\right) \equiv E \otimes_{\AA}^{\tau} F$ is a topological $\mathbb{A}$-algebra of the same type as $E, F$.

See also [14, Definition 1.1]. Moreover, we still assume the following conditions for $\tau$ :

(2.2) the canonical map $E \times F \rightarrow E \otimes_{\mathbb{A}}^{\tau} F$ is separately continuous;

(2.3) for every topological $\mathbb{A}$-algebra $G$ and every pair $(f, g) \in$ $\mathscr{M}_{\mathbb{A}}(E, G) \times \mathscr{M}_{\mathrm{A}}(F, G), \quad f \otimes g \in \mathscr{L}_{\mathbb{A}}\left(E \otimes_{\mathbb{A}}^{\tau} F, G\right)$.

Here $\mathscr{M}_{\mathrm{A}}(E, G)$ denotes the set of non-zero continuous $A$-morphisms of $E$ into $G$ ('generalized spectrum') endowed with the topology induced on it by $\mathscr{L}_{\mathbb{A}}(E, G)_{s}$ (the set of continuous $\mathbb{A}$-linear maps between the corresponding modules equipped with the simple convergence topology). If the algebras involved have identities, the respective morphisms are assumed to be 'identity preserving'. The previous notions constitute a 'vectorization' of the classical concept of the numerical spectrum (Gel'fand space) of a topological algebra $E$ (denoted by $\mathfrak{M}(E) ;[19]$ ). As an example, we note that the projective $\mathbb{A}$-tensor product topology, say $\pi$, provides a compatible $\mathbb{A}$-topology on $E \otimes_{\mathbb{A}} F$, in case of locally convex $\mathbb{A}$-algebras (cf. [14, Definition 5.1]). We come now to the promised assertion, namely, the definition of the topological tensor product $\mathscr{A}$-algebra sheaf:

So let $\mathscr{E}=\left\{\mathscr{E}(U), \varphi_{U}^{V}\right\}, \quad \mathscr{F}=\left\{\mathscr{F}(U), g_{U}^{V}\right\}$ be topological $\mathscr{A}=\left\{\mathscr{A}(U), \sigma_{U}^{V}\right\}-$ algebra sheaves on $X$ with continuous multiplications.

Then, the correspondence

$$
U \mapsto \mathscr{E}(U) \hat{\otimes}_{\mathscr{A}(U)}^{\tau_{U}} \mathscr{F}(U),
$$

with $U \subseteq X$ open and $\tau_{U}$ a compatible $\mathscr{A}(U)$-tensorial topology (cf. (2.1)), is said to be a topological $\mathscr{A}$-algebra presheaf on $X$, if

$$
h_{U}^{V}:=\varphi_{U}^{V} \otimes g_{U}^{V}: \mathscr{E}(V) \hat{\otimes}_{\mathscr{A}(V)}^{\tau_{V}} \mathscr{F}(V) \rightarrow \mathscr{E}(U) \hat{\otimes}_{\mathscr{A}(U)}^{\tau_{U}} \mathscr{F}(U),
$$

is continuous for any $U \subseteq V$ (cf. [16, 20]). The sheaf generated by (2.4) is called the topological tensor product $\mathscr{A}$-algebra sheaf of $\mathscr{E}, \mathscr{F}$ and is denoted by $\mathscr{E} \hat{\otimes}_{\mathscr{A}}^{\tau} \mathscr{F}$. 
The presheaf (2.4) is not, in general, a sheaf. However, this may happen for suitable topological algebra sheaves, as for instance in the following examples.

EXAMPLE 2.1. 1) Let $M, N, \mathbb{A}$ be unital, commutative, nuclear, Fréchet, locally convex algebras with $M, N$ topological $\mathbb{A}$-algebras. Then, for any open $\sigma$-compact subset $U$ of a compact space $X$, one has

$$
\mathscr{C}\left(U, M \hat{\otimes}_{\mathbb{A}} N\right)=\mathscr{C}(U, M) \underset{\mathscr{C}(U, A)}{\hat{\otimes}} \mathscr{C}(U, N)
$$

up to an isomorphism of locally convex $\mathbb{A}$-algebras (cf. [18]). Thus, the correspondence

$$
U \mapsto \mathscr{C}(U, M) \underset{\mathscr{C}(U, \mathbb{A})}{\hat{\otimes}} \mathscr{C}(U, N)
$$

defines a locally convex $\{\mathscr{C}(U, A)\}$-algebra sheaf over $X$.

2) By considering a 2nd-countable compact $\mathscr{C}^{\infty}$-manifold (resp. a Stein manifold), we can replace in the above example continuous functions by $\mathscr{C}^{\infty}$ (resp. holomorphic) ones. See also $[16, \S 4]$ and $[18$, Scholium].

3) We continue by considering another instance where the presheaf (2.4) yields a sheaf. But we still need some more terminology : thus let $A$ be a locally convex algebra and $\mathscr{E}=\left\{\mathscr{E}(U), \varphi_{U}^{V}\right\}$ a locally convex $\mathbb{A}$-algebra sheaf on $X$. Let also $\mathscr{E}_{\mathbb{A}}^{\prime}$ be the $A$-dual pre-co-sheaf on $X$ corresponding to $\mathscr{E}$; that is, we are given a covariant functor

$$
U \mapsto\left(\mathscr{E}(U)_{\mathbb{A}}\right)^{\prime} \equiv \mathscr{L}_{\mathbb{A}}(\mathscr{E}(U), \mathbb{A}),
$$

such that, for any open $U \subseteq V \subseteq X$, one defines as restriction map the transpose of (the restriction map) $\varphi_{U}^{V}$, that is

$$
\tilde{\varphi}_{V}^{U} ;={ }^{t} \varphi_{U}^{V}:\left(\mathscr{E}(U)_{\AA}\right)^{\prime} \rightarrow\left(\mathscr{E}(V)_{\AA}\right)^{\prime}
$$

(cf. also [13, p. 166]). Thus we say that $\mathscr{E}_{\mathbb{A}}^{\prime}$ is $\mathbb{A}-$ flabby in the case that

$$
\left(\mathscr{E}\left(U_{\alpha}\right)_{\mathbb{A}}\right)^{\prime} \rightarrow\left(\mathscr{E}(U)_{\mathrm{A}}\right)^{\prime} \rightarrow 0
$$

is an exact sequence for an open covering $\left(U_{\alpha}\right)_{\alpha}$ of $U \subseteq X$ open (cf. also [20, p. 217, (1.17)]). On the other hand, suppose that the following condition holds :

if $\mathscr{E}$ is a locally convex algebra sheaf on $X$ then, for every open $U \subseteq X$ and an open covering $\left(U_{\alpha}\right)_{\alpha}$ of $U$, one has

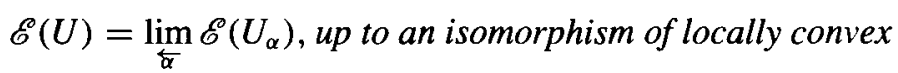
algebras. 
This happens, for instance, in case of the sheaf $\mathscr{C}^{\infty} \equiv\left\{\mathscr{C}^{\infty}(U), U \subseteq Y\right.$ open $\}$ on a finite-dimensional compact $\mathscr{C}^{\infty}$-manifold $Y$, or for the sheaf $\mathscr{O} \equiv\{\mathscr{O}(U), U \subseteq X$ open\} of holomorphic functions on a complex manifold $X$ (cf. $[19,21]$ ).

Now, the following specializes to $[20$, Theorem 2.1] for $\mathbb{A}=\mathbb{C}$; cf. also [22, Lemma 2.1] and [3, Proposition 9.2, Corollary 9.3].

PROPOSITION 2.1. Let $\mathbb{A}$ be a unital locally convex algebra and $\mathscr{E}, \mathscr{F}$ locally convex A-algebra sheaves on $X$ with continuous multiplications such that the sequence

$$
O \rightarrow E(U) \hat{\otimes}_{\mathbb{A}}^{\tau_{U}} \mathscr{F}(U) \rightarrow L_{s}\left(\left(\mathscr{E}(U)_{\mathbb{A}}\right)^{\prime}, \mathscr{F}(U)\right)
$$

is exact, for each open $U \subseteq X$. Moreover, suppose that either one of $\mathscr{E}, \mathscr{F}$ defines an A-flabby pre-co-sheaf such that the canonical projection maps in (2.8) have dense images while the other is an injective $\mathbb{A}$-module sheaf. Then one gets

$$
\mathscr{E}(U) \hat{\otimes}_{\mathrm{A}}^{\tau_{U}} \mathscr{F}(U)=\left(\mathscr{E} \hat{\otimes}_{\mathbb{A}}^{\tau} \mathscr{F}\right)(U),
$$

up to an isomorphism of $\mathbb{A}$-algebras, for every open $U \subseteq X$. In particular, $\mathscr{E} \hat{\otimes}_{\mathbb{A}}^{r} \mathscr{F}$ is a locally convex $\mathbb{A}$-algebra sheaf.

Concerning (2.11), $L_{s}\left(\left(\mathscr{E}(U)_{\mathbb{A}}\right)^{\prime}, \mathscr{F}(U)\right)$ stands for the space of linear maps of $\left(\mathscr{E}(U)_{\mathbb{A}}\right)^{\prime}$ into $\mathscr{F}(U)$ endowed with the simple convergence topology. Such a situation one has, for instance, by taking the projective $\mathbb{A}$-tensor product algebra $\mathscr{E}(U) \hat{\otimes}_{\mathbb{A}}^{\pi} \mathscr{F}(U) \equiv \mathscr{E}(U) \hat{\otimes}_{\mathbb{A}} \mathscr{F}(U)$ of two Fréchet locally convex A-algebra sheaves $\mathscr{E}, \mathscr{F}$ with one of them being nuclear and $\mathbb{A}$ having an identity element (cf. [8], [12, p. 144, (7)], [14, Definition 5.1]).

PROOF OF PROPOSITION 2.1. Suppose $\mathscr{E}$ defines an $\mathbb{A}$-flabby pre-co-sheaf and let $\varphi_{U}^{V}\left(\right.$ resp. $g_{U}^{V}$ ) be the canonical restriction maps of $\mathscr{E}=\{\mathscr{E}(U)\}($ resp. $\mathscr{F}=\{\mathscr{F}(U)\}$ ). By definition of $\mathscr{E} \hat{\otimes}_{\AA}^{\tau} \mathscr{F}$, one gets a (canonical) morphism of $\mathbb{A}$-algebras $\rho: \mathscr{E}(U) \hat{\otimes}_{\AA}^{\tau_{U}}$ $\mathscr{F}(U) \rightarrow\left(\mathscr{E}_{\hat{\mathbb{A}}}^{\tau} \mathscr{F}\right)(U)$, which is a bijection : let $f \in \mathscr{E}(U) \hat{\otimes}_{\AA}^{\tau_{U}} \mathscr{F}(U) \subseteq$ $L_{s}\left(\left(\mathscr{E}(U)_{\AA}\right)^{\prime}, \mathscr{F}(U)\right)$ with $\rho(f)=0$. Then there exists an open covering $\left(U_{\alpha}\right)_{\alpha}$ of $U$ such that $\varphi_{U_{\alpha}}^{U}(f)=0$; that is, for each $x^{\prime} \in\left(\mathscr{E}(U)_{\mathbb{A}}\right)^{\prime}$, one gets $x_{\alpha}^{\prime} \in\left(\mathscr{E}\left(U_{\alpha}\right)_{\mathbb{A}}\right)^{\prime}$ with $x^{\prime}=x_{\alpha}^{\prime} \circ \varphi_{U_{\alpha}}^{U}$ (cf. (2.9)) and hence $\left.f\left(x_{\alpha}^{\prime} \circ \varphi_{U_{\alpha}}^{U}\right)\right|_{U}=\left.f\left(x^{\prime}\right)\right|_{U}=O$, for every $x^{\prime} \in\left(\mathscr{E}(U)_{\AA}\right)^{\prime}$, so that $f=0$. Thus, the map $\rho$ is 1-1. On the other hand, if $f \in\left(\mathscr{E} \hat{\otimes}_{\AA}^{\tau} \mathscr{F}\right)(U)$, there exists an open covering $\mathfrak{N} \equiv\left(U_{\alpha}\right)_{\alpha}$ of $U$ such that, for each $U_{\alpha} \in \mathfrak{N}$, one has $f_{\alpha}=\left.f\right|_{U_{\alpha}}$ with $f_{\alpha} \in L_{s}\left(\left(\mathscr{E}\left(U_{\alpha}\right)_{\mathbb{A}}\right)^{\prime}, \mathscr{F}\left(U_{\alpha}\right)\right)$. Moreover, for any $U_{\alpha}, U_{\beta} \in \mathfrak{N}$ with $U_{\alpha} \subseteq U_{\beta}$, one obtains $g_{U_{\alpha}}^{U_{\beta}} \circ f_{\beta} \circ \tilde{\varphi}_{U_{\beta}}^{U_{\alpha}}=f_{\alpha}$, where $\tilde{\varphi}_{U_{\beta}}^{U_{\alpha}}$ is the transpose of $\varphi_{U_{\alpha}}^{U_{\beta}}$ (cf. also (2.8)). So the family $\left(f_{\alpha}\right)_{\alpha}$ defines an element $s$ in the domain of $\rho$ (cf. also [1, Chapter II, \$\$6.1, 6.2]) such that $\rho(s)=f$; namely, $\rho$ is an onto map as well. Finally, $\mathscr{E} \hat{\otimes}_{\mathbb{A}}^{T} \mathscr{F}$ is made into a sheaf with local section algebras as locally $\mathbb{A}$-algebras, by taking (2.12) as a topological $\mathbb{A}$-isomorphism, for every open $U \subseteq X$. 


\section{The sheaf of morphisms}

In this section, by considering the sheaf of morphisms of a topological tensor product $A$-algebra sheaf into a given (topological) $A$-algebra sheaf, we give a fundamental relation connecting the previous morphisms with those of the factor sheaves (Theorem 3.1). The technique applied is basically functional analytic in nature compared with the standard 'discrete case' (cf., for instance, [2, 25]). The following lemma, needed for the sequel, provides a sample.

LEMMA 3.1. Let $\mathscr{E}, \mathscr{F}, \mathscr{G}$ be unital topological $\mathscr{A}$-algebra sheaves having continuous multiplications, with $\mathscr{G}$ being complete. Moreover, let $\mathscr{E} \hat{\otimes}_{\mathscr{A}}^{\tau} \mathscr{F}$ be the topological tensor product $\mathscr{A}$-algebra sheaf of $\mathscr{E}, \mathscr{F}$ generated by (2.4) such that the topology $\tau_{U}$ satisfies the analogous conditions (2.2), (2.3). Then, one gets the following (canonical) injection between the corresponding sets of topological $\mathscr{A}$-sheaf morphisms:

$$
\operatorname{Hom}_{\mathscr{A}}\left(\mathscr{E} \hat{\otimes}_{\mathscr{A}}^{\tau} \mathscr{F}, \mathscr{G}\right) \stackrel{\subsetneq}{\lessgtr} \operatorname{Hom}_{\mathscr{A}}(\mathscr{E}, \mathscr{G}) \times \operatorname{Hom}_{\mathscr{A}}(\mathscr{F}, \mathscr{G})
$$

In particular, if $\mathscr{G}$ is commutative, one has the bijection

$$
\operatorname{Hom}_{\mathscr{A}}\left(\mathscr{E} \hat{\otimes}_{\mathscr{A}}^{\tau} \mathscr{F}, \mathscr{G}\right)=\operatorname{Hom}_{\mathscr{A}}(\mathscr{E}, \mathscr{G}) \times \operatorname{Hom}_{\mathscr{A}}(\mathscr{F}, \mathscr{G}) .
$$

PROOF. If $h \in \operatorname{Hom}_{\mathscr{A}}\left(\mathscr{E} \hat{\otimes}_{\mathscr{A}}^{\tau} \mathscr{F}, \mathscr{G}\right)$ then by definition, one gets the following family of continuous $\mathscr{A}(U)$-morphisms (see also (2.4))

$$
h_{U}: \mathscr{E}(U) \hat{\otimes}_{\mathscr{A}(U)}^{\tau_{U}} \mathscr{F}(U) \rightarrow \mathscr{G}(U), \quad U \subseteq X \text { open; }
$$

hence (cf. [14, Proposition 3.1]), one obtains the (continuous $\mathscr{A}(U)$-)morphisms;

$$
\begin{gathered}
f_{U}: \mathscr{E}(U) \rightarrow \mathscr{G}(U): s \mapsto f_{U}(s):=h_{U}\left(s \otimes 1_{\mathscr{F}}\right), \\
g_{U}: \mathscr{F}(U) \rightarrow \mathscr{G}(U): t \mapsto g_{U}(t):=h_{U}\left(1_{\mathscr{E}} \otimes t\right),
\end{gathered}
$$

with $U \subseteq X$ open, and $1_{\mathscr{E}}, 1_{\mathscr{F}}$ being the identities of $\mathscr{E}(U), \mathscr{F}(U)$ respectively. So if $f: \mathscr{E} \rightarrow \mathscr{G}, \quad g: \mathscr{F} \rightarrow \mathscr{G}$ are the corresponding topological $\mathscr{A}$-sheaf morphisms (cf. $\S 1$ and also $[4$, p. 32]), one obtains the map

$$
\begin{aligned}
\Phi: \operatorname{Hom}_{\mathscr{A}}\left(\mathscr{E} \hat{\otimes}_{\mathscr{A}}^{\tau} \mathscr{F}, \mathscr{G}\right) & \rightarrow \operatorname{Hom}_{\mathscr{A}}(\mathscr{E}, \mathscr{G}) \times \operatorname{Hom}_{\mathscr{A}}(\mathscr{F}, \mathscr{G}): \\
h & \mapsto \Phi(h):=(f, g)
\end{aligned}
$$

which is an injection. The assertion follows, by checking it 'stalkwise' (see, for example, [25, p. 16, Proposition 1.10]), based on [14, Proposition 3.1, (3.7)],[16, 
Theorem 3.2]. On the other hand, if $\mathscr{G}$ is commutative and $(f, g) \in \operatorname{Hom}_{\mathscr{A}}(\mathscr{E}, \mathscr{G}) \times$ $\operatorname{Hom}_{\mathscr{A}}(\mathscr{F}, \mathscr{G})$, one gets the following continuous $A(U)$-morphisms (see also (3.3))

$$
h_{U} \equiv f_{U} \hat{\otimes} g_{U}: \mathscr{E}(U) \hat{\otimes}_{\mathscr{A}(U)}^{\tau_{U}} \mathscr{F}(U) \rightarrow \mathscr{G}(U), \quad U \subseteq X \text { open. }
$$

So one obtains a unique topological $\mathscr{A}$-sheaf morphism $h: \mathscr{E} \hat{\otimes}_{\mathscr{A}}^{r} \mathscr{F} \rightarrow \mathscr{G}$ (cf. [4, p. 38]) such that $\Phi(h)=(f, g)$ (cf. (3.5), (3.6)).

By considering the sheaves of Lemma 3.1 restricted on an open $U \subseteq X$ one gets the following $\left.\mathscr{A}\right|_{U}$-sheaf isomorphism

$$
\left.\left(\mathscr{E} \hat{\otimes}_{\mathscr{A}}^{\tau} \mathscr{F}\right)\right|_{U}=\left.\left.\mathscr{E}\right|_{U} \hat{\otimes}_{\left.\mathscr{A}\right|_{U}}^{\tau} \mathscr{F}\right|_{U}
$$

(cf. (2.4) and [4, p. 38]). In this regard, if $\mathscr{E}, \mathscr{F}$ are topological $\mathscr{A}$-algebra sheaves, we consider the sheaf

$$
\mathscr{H}_{o m_{\mathscr{A}}}(\mathscr{E}, \mathscr{F})=\left\{\operatorname{Hom}_{\left.\mathscr{A}\right|_{U}}\left(\left.\mathscr{E}\right|_{U},\left.\mathscr{F}\right|_{U}\right), U \subseteq X \text { open }\right\}
$$

of topological $\mathscr{A}$-sheaf morphisms of $\mathscr{E}$ into $\mathscr{F}$ over $X$ (cf. also $[2,25])$. So we are in a position to state the following basic results.

THEOREM 3.1. Under the hypotheses of Lemma 3.1, one has the following sheaf monomorphism

$$
\mathscr{H} o m_{\mathscr{A}}\left(\mathscr{E} \hat{\otimes}_{\mathscr{A}}^{\tau} \mathscr{F}, \mathscr{G}\right) \subsetneq \mathscr{H} o m_{\mathscr{A}}(\mathscr{E}, \mathscr{G}) \times \mathscr{H} o m_{\mathscr{A}}(\mathscr{F}, \mathscr{G}) .
$$

In particular, if $\mathscr{G}$ is commutative one obtains the sheaf isomorphism:

$$
\mathscr{H} \operatorname{om}_{\mathscr{A}}\left(\mathscr{E} \hat{\otimes}_{\mathscr{A}}^{\tau} \mathscr{F}, \mathscr{G}\right)=\mathscr{H} o m_{\mathscr{A}}(\mathscr{E}, \mathscr{G}) \times \mathscr{H}_{o m_{\mathscr{A}}}(\mathscr{F}, \mathscr{G}) .
$$

PROOF. The assertion follows from (3.1), (3.2), (3.7), (3.8) by applying standard arguments (see also, for example, [25, p. 22, Theorem 4.2]).

\section{Structure sheaf envelopes}

Given a topological algebra sheaf $\mathscr{E}$ over $X$, the structure sheaf envelope $\mathbf{H}(X, \mathscr{E})$ of the pair $(X, \mathscr{E})$ (the so-called topological geometric space) considered by A. Mallios $[22$, p. $374,(2.1)]$, is defined via the (numerical) spectrum (Gel'fand space) of the topological algebra $\mathscr{E}(X) \equiv \Gamma(X, \mathscr{E})$ of global sections of $\mathscr{E}$. Thus one sets, by definition,

$$
\mathbf{H}(X, \mathscr{E}):=\mathfrak{M}(\mathscr{E}(X))
$$


More generally, based on (1.1), an analogous relation to (4.1) can be displayed for any subset $S \subseteq X$. The motivation to the above is, of course, the envelope of holomorphy of a complex analytic space $(X, \mathscr{O})$ defined as the (numerical) spectrum of the topological algebra $\mathscr{O}(X)$ (cf., for instance, [10] or [19, Chapter V, (4.15)]). Below we are interested in the form, which takes the structure sheaf envelope in case of a topological tensor product $\mathscr{A}$-algebra sheaf.

Thus, let $\mathscr{E}, \mathscr{F}$ be locally convex $\mathscr{A}$-algebra sheaves over $X$ with continuous multiplications. Moreover, assume that the presheaf

$$
U \mapsto \mathscr{E}(U) \underset{\mathscr{A}(U)}{\hat{\otimes}} \mathscr{F}(U), \quad U \subseteq X \text { open, }
$$

is also a sheaf (cf. Examples 2.1). Here the second member of (4.2) stands for the complete projective $\mathscr{A}(U)$-tensor product (algebra) of $\mathscr{E}(U), \mathscr{F}(U)$ (cf. (2.4) for $\tau=\pi)$. Thus, one has, according to our hypothesis, that

$$
\left(\mathscr{E} \hat{\otimes}_{\mathscr{A}} \mathscr{F}\right)(U)=\mathscr{E}(U) \underset{\mathscr{A}(U)}{\hat{\otimes}} \mathscr{F}(U),
$$

within an isomorphism of locally convex $\mathscr{A}(U)$-algebras, for every open $U \subseteq X$.

PROPOSITION 4.1. Let $\mathscr{E}, \mathscr{F}$ be unital locally convex $\mathscr{A}$-algebra sheaves over $X$ with continuous multiplications and locally equicontinuous spectra such that (4.3) is satisfied. Then, for any $S \subseteq X$, one gets

$$
\mathbf{H}\left(S, \mathscr{E} \hat{\otimes}_{\mathscr{A}} \mathscr{F}\right)=\mathbf{H}(S, \mathscr{E}) \underset{\mathbf{H}(S, \mathscr{A})}{\times} \mathbf{H}(S, \mathscr{F}),
$$

up to a homeomorphism.

(In the second member of (4.4) we mean the pull-back of the corresponding topological spaces).

PROOF. By (1.1) and (4.3)

$$
\left(\mathscr{E} \hat{\otimes}_{\mathscr{A}} \mathscr{F}\right)(S)=\lim _{U \supseteq \mathfrak{S}}(\mathscr{E}(U) \hat{\mathscr{Q}(U)} \mathscr{F}(U))
$$

is a unital topological $\mathscr{A}(S)$-algebra with continuous multiplication. Moreover, one gets

$$
\begin{aligned}
\mathfrak{M}\left(\left(\mathscr{E} \hat{\otimes}_{\mathscr{A}} \mathscr{F}\right)(S)\right) & =\lim _{\overleftarrow{U} \boldsymbol{S}} \mathfrak{M}(\mathscr{E}(U) \underset{\mathscr{A}(U)}{\hat{\otimes}} \mathscr{F}(U))=\lim _{\overleftarrow{U}(\mathfrak{S}}(\mathfrak{M}(\mathscr{E}(U)) \underset{\mathfrak{M}(\mathscr{A}(U))}{\times} \mathfrak{M}(\mathscr{F}(U)) \\
& =\mathfrak{M}(\mathscr{E}(S)) \underset{\mathfrak{M}(\mathscr{A}(S))}{\times} \mathfrak{M}(\mathscr{F}(S))
\end{aligned}
$$

where the equalities denote homeomorphisms (cf. [14, Theorem 2.1], [16, Theorem 2.1] and [19, Chapter V, Lemma 2.2, Theorem 3.1; Chapter XII, Theorem 1.2]). The assertion is now an immediate consequence of (4.1). 
The relation (4.4) takes a more convenient form by considering a particular class of topological algebra sheaves and a suitable subspace $S$ of $X$. First, a topological algebra sheaf $\mathscr{E}$ over $X$ is said to be a topological function algebra sheaf if $\mathscr{E}$ is a subsheaf of the $\mathscr{C}_{X}$ of germs of $\mathbb{C}$-valued continuous functions on $X$ (cf. [22, p. 373]). Thus the (topological algebra) sheaf $\mathscr{C}_{X}^{\infty}$ of $\mathbb{C}$-valued $\mathscr{C}^{\infty}$-functions on a real manifold $X$ (cf. [19, Chapter IV, Example 4.(2)]) and the (topological algebra) sheaf $\mathscr{O}$ of holomorphic functions on a given complex (analytic) manifold [ibid., Example 4.(3)] are important instances of such sheaves.

Moreover, given a topological algebra sheaf $\mathscr{E}$ over $X$, we say that an $S \subseteq X$ is $\mathscr{E}$-convex, whenever the relation

$$
\mathfrak{M}(\mathscr{E}(S))=S
$$

is valid, within a homeomorphism (cf. [21, p. 376] or [22, p. 377, (3.1)]). This is true, for instance, in case of a Stein manifold $(X, \mathscr{O})$ and a compact holomorphically convex subset $S$ of $X$ (cf. $[9,10]$ ).

Thus, we are now in the position to state the next corollary whose proof follows straightforwardly from Proposition 4.1 and (4.5).

COROLLARY 4.1. Suppose that the hypotheses of Proposition 4.1 are satisfied, where the corresponding sheaves are topological function algebra sheaves. Then $S$ is $\mathscr{E} \hat{\otimes}_{\mathscr{A}} \mathscr{F}$-convex if it is an $\mathscr{E}, \mathscr{F}$ and $\mathscr{A}$-convex subset of $X$. In that case one gets, in particular, the following homeomorphism

$$
\mathbf{H}\left(S, \mathscr{E} \hat{\otimes}_{\mathscr{A}} \mathscr{F}\right)=S .
$$

\section{Generalized structure sheaf envelopes}

We extend below the results of the previous section to generalized structure sheaf envelopes. Namely, one substitutes a suitable topological algebra for $\mathbb{C}$ in the second member of (4.1), while the given topological algebra sheaf $\mathscr{E}$ may have coefficients from the (algebra of) global sections of the 'sheaf of coefficients' $\mathscr{A}$. More precisely, in addition to the given pair, $(X, \mathscr{E})$ as above, we consider a topological $\mathscr{A}(S)$-algebra $G$ for a given $S \subseteq X$. Thus, one defines the generalized structure sheaf envelope of $S$ with respect to $G$ by the relation (cf. also $\S 2$ )

$$
\mathbf{H}_{\mathscr{A}}^{G}(S, \mathscr{E}):=\mathscr{M}_{\mathscr{A}(S)}(\mathscr{E}(S), G)
$$

that is, the generalized $\mathscr{A}(S)$-spectrum of the topological $\mathscr{A}(S)$-algebra $\mathscr{E}(S)$ relative to $G$ (cf. $[14, \S 3]$ ). Concerning the topological algebras $\mathscr{A}(S), \mathscr{E}(S)$ see (1.1). 
Henceforth we assume that the algebras involved are unital. So let

$$
\mathscr{M}_{(\mathscr{A}(U), \mathscr{A}(S))}(\mathscr{E}(U), G)
$$

be the set of continuous $(\mathscr{A}(U), \mathscr{A}(S))$-morphisms of $\mathscr{E}(U)$ into $G$. By the last term one means the continuous morphisms of $\mathscr{E}(U)$ into $G$ with respect to the (canonical) connecting maps $\sigma_{U}: \mathscr{A}(U) \rightarrow \mathscr{A}(S)$ (see (1.1) and [16, (1.1)]). Moreover, the same set is endowed with the topology of simple convergence in $\mathscr{E}(U)$. The resulted topological space is called the $(\mathscr{A}(U), \mathscr{A}(S))$-spectrum (of $\mathscr{E}(U)$ with respect to $G$ ). Thus, we have the next homeomorphism (cf. also [16, (3.1)])

$$
\mathscr{M}_{\mathscr{A}(S)}(\mathscr{E}(S), G)=\lim _{\overleftarrow{U} \underline{S}} \mathscr{M}_{(\mathscr{A}(U), \mathscr{A}(S))}(\mathscr{E}(U), G)
$$

Now, let $\mathscr{E}, \mathscr{F}$ be topological $\mathscr{A}$-algebra sheaves, $U$ an element of a fundamental system of neighborhoods of $S$ and

$$
\begin{aligned}
Q(U) & :=\left\{\left(s_{U}, t_{U}\right): s_{U}\left(x_{U}\right) t_{U}\left(y_{U}\right)=t_{U}\left(y_{U}\right) s_{U}\left(x_{U}\right), \quad x_{U} \in \mathscr{E}(U), y_{U} \in \mathscr{F}(U)\right\} \\
& \subseteq \mathscr{M}_{(\mathscr{A}(U), \mathscr{A}(S))}(\mathscr{E}(U), G) \times \mathscr{M}_{(\mathscr{A}(U), \mathscr{A}(S))}(\mathscr{F}(U), G),
\end{aligned}
$$

a closed subset. Hence, if

$$
\begin{aligned}
Q(S) & :=\{(s, t): s(x) t(y)=t(y) s(x), \quad x \in \mathscr{E}(S), y \in \mathscr{F}(S)\} \\
& \subseteq \mathscr{M}_{\mathscr{A}(S)}(\mathscr{E}(S), G) \times \mathscr{M}_{\mathscr{A}(S)}(\mathscr{F}(S), G),
\end{aligned}
$$

still a closed subset, one gets

$$
Q(S)=\lim _{\overleftarrow{U} \underline{S} S} Q(U)
$$

within a homeomorphism. See [14, Theorem 3.1], [16, (3.3)].

THEOREM 5.1. Let $\mathscr{E}, \mathscr{F}$ be unital locally convex $\mathscr{A}$-algebra sheaves over X having continuous multiplications and locally equicontinuous $(\mathscr{A}(U), \mathscr{A}(S))$-spectra (cf. (5.2)) relative to a complete locally convex $\mathscr{A}(S)$-algebra $G$ which also has continuous multiplication. Moreover, let (4.3) be satisfied. Then

$$
\mathbf{H}_{\mathscr{A}}^{G}\left(S, \mathscr{E}_{\mathscr{\otimes}} \mathscr{F}\right) \cong Q(S) \subseteq \mathbf{H}_{\mathscr{A}}^{G}(S, \mathscr{E}) \times \mathbf{H}_{\mathscr{A}}^{G}(S, \mathscr{F}),
$$

up to a homeomorphism (cf. (5.5)). In particular, if $G$ is commutative, one has the homeomorphism

$$
\mathbf{H}_{\mathscr{A}}^{G}\left(S, \mathscr{E} \hat{\otimes}_{\mathscr{A}} \mathscr{F}\right)=\mathbf{H}_{\mathscr{A}}^{G}(S, \mathscr{E}) \times \mathbf{H}_{\mathscr{A}}^{G}(S, \mathscr{F})
$$


PROOF. By hypothesis, $\mathscr{M}_{(\mathscr{A}(U), \mathscr{A}(S))}\left(\mathscr{E}(U) \hat{\otimes}_{\mathscr{A}(U)} \mathscr{F}(U), G\right)$ is locally equicontinuous. Therefore, in view of (5.3), (5.5) one has the following homeomorphisms (see also [14, Theorem 3.1], [16, Theorem 3.2] and [19, Chapter V, p. 180, 8(3); Chapter XII, §4]).

$$
\begin{aligned}
\mathscr{M}_{\mathscr{A}(S)}\left(\left(\mathscr{E} \hat{\otimes}_{\mathscr{A}} \mathscr{F}\right)(S), G\right) & =\lim _{U \supseteq S} \mathscr{M}_{(\mathscr{A}(U), \mathscr{A}(S))}\left(\left(\mathscr{E} \hat{\otimes}_{\mathscr{A}} \mathscr{F}\right)(U), G\right) \\
& =\lim _{\overleftarrow{U}} \mathscr{M}_{(\mathscr{A}(U), \mathscr{A}(S))}\left(\mathscr{E}(U) \hat{\otimes}_{\mathscr{A}(U)} \mathscr{F}(U), G\right) \\
& =\lim _{\overleftarrow{U}} Q(U)=Q(S)
\end{aligned}
$$

So (5.6), (5.7) are immediate consequences of (5.1).

\section{REMARKS 5.1.}

1) We note here, in passing, that (3.9), (3.10) could be obtained by applying Theorem 5.1, when assuming suitable supplementry conditions on the $\mathscr{A}$-algebra sheaves involved. We may suppose, for instance, that these sheaves satisfy (2.10).

2) The preceding relations (5.6), (5.7) are also true in case $G$ is the algebra of germs of a topological $\mathscr{A}$-algebra sheaf $\mathscr{G}$ over an arbitrary subset $S$ of $X, \mathscr{G}(S)$, when the latter algebra has the corresponding properties of $G$. Concerning the completeness of $\mathscr{G}(S)$ this, of course, could be provided for suitable algebras $\mathscr{G}(U)$, with $U \supseteq S$. See, for instance, [19, Chapter VIII, §9.(1)].

3) The results of this section are also true by replacing the projective (tensorial) topology $\pi$ by a more general (compatible $\mathbb{A}$-) topology $\tau$, satisfying analogous conditions to those needed for the decompositions of the spectra of topological tensor product $\mathbb{A}$-algebras (cf. (2.2), (2.3) and also [14, (3.2), (3.3)]).

\section{A Dirac transform}

We consider in this section an important class of morphisms, the continuous central morphisms defined, in particular, on the topological $\mathscr{A}(S)$-algebra $\left(\mathscr{E} \hat{\otimes}_{\mathscr{A}} \mathscr{F}\right)(S)$. As a main application one gets an expression of any such morphism through a map which by an extension of the classical case, one might call a Dirac transform (Theorem 6.1).

In this regard, A. Mallios [22, p. 378] (see also [21, pp. 358, 359]) considered the notion of a continuous ( $\mathbb{C}$-) central morphism $h: E \rightarrow F$ between two topological algebras $E, F$, such that $\operatorname{Im}(h)$ has a trivial centre over $\mathbb{C}$ (central $\mathbb{C}$-algebra); that is, $\operatorname{Im}(h) \cap(\operatorname{Im}(h))^{\prime}=\mathbb{C} \cdot 1_{F}$, where $(\operatorname{Im}(h))^{\prime}=\{y \in F: y \cdot h(x)=h(x) \cdot y, x \in E\}$ is the commutant of $\operatorname{Im}(h)$ and $1_{F}$ the identity of $F$.

Now, if $h$ is a continuous $\mathbb{A}$-morphism between two topological $\mathbb{A}$-algebras $E, F$, then one defines a continuous $\mathbb{A}$-central $(\mathbb{A}$-)morphism, where now $\operatorname{Im}(h)$ is a central 
$\mathbb{A}$-algebra, that is it has a trivial center over $\mathbb{A}(\mathrm{cf} .[14,17])$. Thus, a continuous (C-)central $\mathbb{A}$-morphism is also an $\mathbb{A}$-central $(\mathbb{A}$-) morphism. We denote by

$$
\mathscr{M}_{\AA}^{\mathbb{C}}(E, F)
$$

the set of non-zero continuous $\mathbb{C}$-central $\mathbb{A}$-morphisms of $E$ into $F$ endowed with the relative topology from $\mathscr{M}_{\AA}(E, F)$.

PROPOSITION 6.1. Let $\mathscr{E}, \mathscr{F}$ be unital locally convex $\mathscr{A}$-algebra sheaves over $X$ having continuous multiplications and locally equicontinuous $(\mathscr{A}(U), \mathscr{A}(S))$-spectra with respect to a complete locally convex $\mathscr{A}(S)$-algebra $G$ with continuous multiplications, where $S \subseteq X$. Moreover let (4.3) be satisfied. Then there exists a continuous injection

$$
\mathscr{M}_{\mathscr{A}(S)}^{\mathbb{C}}\left(\left(\mathscr{E} \hat{\otimes}_{\mathscr{A}} \mathscr{F}\right)(S), G\right) \stackrel{\complement}{\rightarrow} \mathscr{M}_{\mathscr{A}(S)}^{\mathbb{C}}(\mathscr{E}(S), G) \times \mathscr{M}_{\mathscr{A}(S)}^{\mathbb{C}}(\mathscr{F}(S), G)
$$

between the corresponding spaces. In particular, if $\mathscr{E}$ is a commutative algebra sheaf, then one has the continuous injection

$$
\mathscr{M}_{\mathscr{A}(S)}^{\mathbb{C}}\left(\left(\mathscr{E} \hat{\otimes}_{\mathscr{A}} \mathscr{F}\right)(S), G\right) \stackrel{\complement}{\rightarrow}(\mathscr{E}(S)) \times \mathscr{M}_{\mathscr{A}(S)}^{\mathbb{C}}(\mathscr{F}(S), G) .
$$

PROOF. By the definitions, one gets the following continuous injection (cf. also [17]);

$$
\mathscr{M}_{\mathscr{A}(S)}^{\mathbb{C}}\left(\lim _{U \supseteq \mathcal{S}}\left(\mathscr{E}(U) \hat{\otimes}_{\mathscr{A}(U)} \mathscr{F}(U)\right), G\right) \stackrel{\subsetneq}{\rightarrow} \mathscr{M}_{\mathscr{A}(S)}^{\mathbb{C}}\left(\lim _{\vec{U}}\left(\mathscr{E}(U) \otimes_{\mathscr{A}(U)}^{\pi} \mathscr{F}(U)\right), G\right) .
$$

Moreover, one has

$$
\mathscr{M}_{\mathscr{A}(S)}^{\mathbb{C}}\left(\lim _{\vec{U}}\left(\mathscr{E}(U) \otimes_{\mathscr{A}(U)}^{\pi} \mathscr{F}(U)\right), G\right)=\mathscr{M}_{\mathscr{A}(S)}^{\mathbb{C}}(\mathscr{E}(S), G) \times \mathscr{M}_{\mathscr{A}(S)}^{\mathbb{C}}(\mathscr{F}(S), G),
$$

up to a homeomorphism (cf. (5.8) and also $[14,17]$ ), so that one gets (6.2). The commutativity of $\mathscr{E}$ entails the commutativity of $\mathscr{E}(S)$ and hence $\mathscr{M}_{\mathscr{S}(S)}^{\mathbb{C}}(\mathscr{E}(S), G)=$ $\mathfrak{M}(\mathscr{E}(S)$ ), within a homeomorphism (cf. also [17]). So (6.3) is an immediate consequence of (6.2) and the last relation.

Under the same hypothesis as for (6.2), let also $\mathscr{E}$ be a topological function $\mathbb{A}$ algebra sheaf over $X$ and $S$ an $\mathscr{E}$-convex subset of $X$ (cf. (4.5)). Thus, in the particular case that $\mathscr{A}$ is the constant sheaf $\mathbb{A}$, then in view of (6.3), (4.5) (cf. also (4.2) and Proposition 2.1) one obtains the continuous injection

$$
\mathscr{M}_{\AA}^{\mathbb{C}}\left(\left(\mathscr{E} \hat{\otimes}_{\AA} \mathscr{F}\right)(S), G\right) \stackrel{\subseteq}{\rightarrow} \times \mathscr{M}_{\AA}^{\mathbb{C}}(\mathscr{F}(S), G) .
$$

In this respect, suppose that the generalized spectra of the algebras $\mathscr{E}(U), \mathscr{F}(U)$ with respect to $G$ (cf. (5.2)) are locally equicontinuous while for all but finite many $U$ s the 
corresponding spectra are equicontinuous. Then, the injection maps (6.2), (6.3), (6.4) are, in particular, homeomorphisms into.

On the other hand, let $\mathbb{A}, F$ be unital locally convex $\mathbb{A}$-algebras with continuous multiplications and $\mathscr{E}$ a unital complete locally convex function $\mathbb{A}$-algebra sheaf over $X$ with continuous multiplication. Moreover, assume that, for any open $U \subseteq X$, the following isomorphism of locally convex $\mathbb{A}$-algebras holds true;

$$
\left(\mathscr{E} \hat{\otimes}_{\mathscr{A}} F\right)(U)=\mathscr{E}(U) \hat{\otimes}_{\mathscr{A}} F .
$$

See also (4.2), (4.3) and Examples 2.1 with $\mathscr{F}, \mathscr{A}$ being the constant sheaves $F, \mathbb{A}$, respectively. Then, if $S \subseteq X$, suppose that

$$
\left(\mathscr{E} \hat{\otimes}_{\mathbb{A}} F\right)(S) \stackrel{\complement}{\hookrightarrow} \mathscr{C}_{c}(S, F)
$$

within a homeomorphism into.

EXAMPLE 6.1. Consider a 2nd-countable space $X$ and closed $S \subseteq X$, admitting a denumerable fundamental system of open $\sigma$-compact neighborhoods. Moreover, suppose that $F$ is a unital (DF)-nuclear locally convex $\mathbb{A}$-algebra (cf. $[14,19])$.

Now, consider the following continuous map (Dirac transform)

$$
\delta: S \rightarrow \mathscr{M}_{\mathbb{A}}\left(\left(\mathscr{E} \hat{\otimes}_{\mathbb{A}} F\right)(S), F\right): x \mapsto \delta(x) \equiv \delta_{x}\left(: f \mapsto \delta_{x}(f):=f(x)\right)
$$

(for an analogous situation of [21, p. 376] and also [22, Theorem 3.1]). Thus we have the following

THEOREM 6.2. Let $\mathscr{E}$ be a unital, complete, locally convex, function $\mathbb{A}$-algebra sheaf over a topological space $X$, having continuous multiplication and locally equicontinuous generalized $\mathbb{A}$-spectrum (cf. (5.1)) with respect to a unital complete locally convex $\mathbb{A}$-algebra $G$ having continuous multiplication. Moreover, let $F$ be a unital, complete, locally convex $\mathbb{A}$-algebra with continuous multiplication and locally equicontinuous $\mathscr{M}_{\mathrm{A}}(F, G)$. Finally, let $S$ be an $\mathscr{E}$-convex subset of $X$ such that the relations (6.5), (6.6) are valid. Then every $h \in \mathscr{M}_{\AA}^{\mathbb{C}}\left(\left(\mathscr{E} \hat{\otimes}_{\mathbb{A}} F\right)(S), G\right)$ has the form

$$
h=\rho \circ \delta_{x}
$$

with $x \in S$ and $\rho \in \mathscr{M}_{\AA}^{\mathbb{C}}(F, G)$.

PROOF. Let $f \in\left(\mathscr{E} \hat{\otimes}_{\mathbb{A}} F\right)(S)$ such that $f=u \otimes y, u \in \mathscr{E}(S), y \in F$. Then, if $\chi \in \mathfrak{M}(\mathscr{E}(S))$ and $1_{F}$ is the identity of $F$, the corresponding relation of (6.3), yields;

$$
\left(\chi \otimes 1_{F}\right)(f)=\left(\chi \otimes 1_{F}\right)(u \otimes y)=\chi(u) \cdot y=u(x) \cdot y=\delta_{x}(f) .
$$


For the third equality see (4.4), for the last equality see (5.6); also see $[14,16]$. Hence, for $f$ as above, $\chi \otimes 1_{F}=\delta_{x}$, such that by linearity and continuity of the preceding maps, for each $h \in \mathscr{M}_{\AA}^{\mathbb{C}}\left(\left(\mathscr{E} \hat{\otimes}_{\AA} F\right)(S), G\right)$, one obtains

$$
h=\overline{\rho \circ\left(\chi \otimes 1_{F}\right)}=\rho \circ \overline{\left(\chi \otimes 1_{F}\right)}=\rho \circ \bar{\delta}_{x}=\rho \circ \delta_{x}
$$

(“-” means continuous extensions; cf. also (6.4), (6.6)).

NOTE. One can get an analogous formula to (6.8) by considering instead of the algebra $F$ a (topological) algebra sheaf $\mathscr{F}$ such that its local section algebras have the same properties as $F$.

\section{References}

[1] N. Bourbaki, Algebra I, Chapters 1-3 (Addison-Wesley, Reading, 1974).

[2] G. E. Bredon, Sheaf theory (McGraw-Hill, New York, 1967).

[3] L. Bungart, 'Holomorphic functions with values in locally convex spaces and applications to integral formulas', Trans. Amer. Math. Soc. 111 (1964), 317-344.

[4] C. H. Dowker, Lectures on sheaf theory (Tata Institute, Bombay, 1956).

[5] J. Dugundji, Topology (Allyn and Bacon, Boston, 1966).

[6] B. R. Gelbaum, 'Tensor products over Banach algebras', Trans. Amer. Math. Soc. 118 (1965), 525-547.

[7] R. Godement, Théorie des faisceaux (Hermann, Paris, 1964).

[8] A. Grothendieck, Produits tensorielles topologiques et espaces nucléaires, Mem. Amer. Math. Soc. 16 (Amer. Math. Soc., Providence, 1955).

[9] R. Gunning and H. Rossi, Analytic functions of several complex variables (Prentice-Hall, Englewood Cliffs, 1965).

[10] R. Harvey and R. O. Wells, Jr., 'Compact holomorphically convex subsets of a Stein manifold', Trans. Amer. Math. Soc. 136 (1969), 509-516.

[11] J. Horvath, Topological vector spaces and distributions (Addison-Wesley, Reading, 1966).

[12] G. Köthe, Topological vector spaces, II (Springer, Berlin, 1979).

[13] A. Kultze, 'Lokalholomorphe Funktionen und das Geschlecht kompakter Riemannschen Flächen', Math. Ann. 143 (1961), 163-186.

[14] A. Kyriazis, 'On the spectra of topological A-tensor product A-algebras', Yokohama Math. J. 31 (1983), 47-65.

[15] _ - 'Tensor products of function algebras', Bull. Austral. Math. Soc. 36 (1987), 417-423.

[16] - 'Direct limits and tensor products of topological A-algebras', Mathematica Japonica 39(1) (1994), 29-41.

[17] —, 'On central morphisms', Bul. Greek Math. Soc., 34 (1992), 45-58.

[18] - 'On tensor product $\alpha$-algebra bundles', in: Proc. Intern. Conf. Advances in the theory of Fréchet spaces, Istanbul, August, 1988, NATO ASI Series C 287 (Kluwer Academic Publishers, Dordrecht, 1989) pp. 223-234.

[19] A. Mallios, Topological algebras: Selected topics (North-Holland, Amsterdam, 1986).

[20] - 'On topological algebra sheaves of a nuclear type', Studia Math. 38 (1970), 215-220. 
[21] — - 'Topological algebras in several complex variables', in: Proc. Intern. Conf. Funct. Anal. and Appl. Madras, 1973, Lecture Notes in Math. 399 (Springer, Berlin, 1974) pp. 342-377.

[22] — , 'Generalised structure sheaf envelopes of topological function algebra spaces', Praktika Akad. Athēnōn 49 (1974), 373-385.

[23] C. E. Rickart, Holomorphic convexity for general function algebras (Yale University, Yale, 1967).

[24] - Natural function algebras (Springer, Berlin, 1979).

[25] B. R. Tennison, Sheaf theory (Cambridge University Press, Cambridge, 1975).

Department of Mathematics

University of Athens

Panepistimiopolis

Athens 15784

Greece 